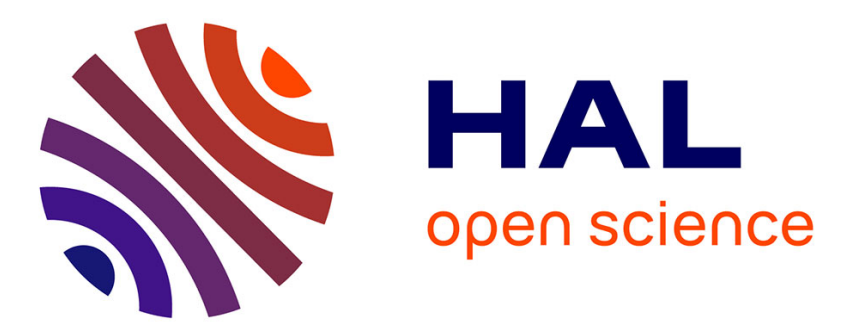

\title{
Digital Resource Semantic Management of Islamic Buildings Case Study on Isfahan Islamic Architecture Digital Collection
}

Tomoaki Okamura, Naoko Fukami, Charles Robert, Frederic Andres

\section{- To cite this version:}

Tomoaki Okamura, Naoko Fukami, Charles Robert, Frederic Andres. Digital Resource Semantic Management of Islamic Buildings Case Study on Isfahan Islamic Architecture Digital Collection. International Journal of Architectural Computing, 2007. hal-03328698

\section{HAL Id: hal-03328698 \\ https://inria.hal.science/hal-03328698}

Submitted on 30 Aug 2021

HAL is a multi-disciplinary open access archive for the deposit and dissemination of scientific research documents, whether they are published or not. The documents may come from teaching and research institutions in France or abroad, or from public or private research centers.
L'archive ouverte pluridisciplinaire HAL, est destinée au dépôt et à la diffusion de documents scientifiques de niveau recherche, publiés ou non, émanant des établissements d'enseignement et de recherche français ou étrangers, des laboratoires publics ou privés. 


\section{Digital Resource Semantic Management} of Islamic Buildings Case Study on Isfahan Islamic Architecture Digital Collection

Tomoaki Okamura, Naoko Fukami, Charles Robert and Frederic Andres

This article describes an innovative way to facilitate and to provide a collaborative semantic management of digital resources of Islamic historical buildings. The approach combines a topic maps-based semantic support to the 5 WIH model (Where, Who, When, What, Who and How) with objective of enabling comprehensive semantics of digital contents for research and education purposes. The topic mapsbased semantic support enables to reduce the problems of semantic gaps among different communities (cultural, linguistic ambiguities among various types of multi-disciplinary experts).

Furthermore, a multi-faceted resource category management, applied to metadata sets and related semantic features, allows metadata optimization of the description of the cultural resources. This innovative approach has been used to build the Isfahan Islamic Architecture Database (IIAD) Collection as a case study. The research demonstrates that a topic mapsbased semantic model applied to collaborative metadata management paradigms can be easily exploited as a tool to enhance traditional architectural and cross-disciplinary studies.

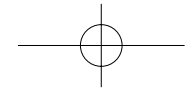




\section{Introduction}

Following the evolution of architectural archives accessible over Internet, new requirements emerged related to semantic understanding of multilingual and multi-disciplinary cultural contents to enhance Islamic Architecture studies. One key issue for metadata management systems is the way to manage semantic interoperability (semantic exchange according to various scope and fields) in architecture field. Various kinds of vocabularies and terminology have been especially used in the description of architecture objects for research and education purposes. In the field of architecture studies, digital resource management system can operate as a repository of digital resources of buildings identified using different metadata records. Furthermore, sophisticated digital archives have evolved to process objects in a dynamic way to extract more semantic (e.g. relation semantic between objects). Multiple metadata points of view can be added or combined according to the profile of end-users (e.g. scholar, architect, historian, students). It is a way to customize the digital resource access using enriched metadata information.

The key reasons of applying semantic architectural computing is the requirement to improve the way to understand historical buildings in relationship with their context, to share the knowledge among communities as buildings express the past in their own existing space. As it is not possible to make the historical building at once, it is important for architectural historians, each building has its own context, its own history, it is already to know our own and also the related world culture. It includes various categories of semantics (e.g. visual semantic, textual semantic) as it has been defined in [37].

In the field of history of Islamic Architecture, a lot of researches have been focused on area from Spain to China, from 7th century to now, very wide range of geographical area and time span [2, 5, 13]. At the same time, Islamic architecture can be defined as objects that Muslim built and used.

Let us take the example of relationship between religion thoughts about mosque, especially the case of the Friday Mosque of Isfahan. In the early Muslim world, the biggest, oldest and most famous mosque in the city made the Friday's noon pray to congregate all of the adult male members of the city and the name of Khalifa (the head of the Muslim world) was recited. Mosque is an English term coming from Spanish mesquite. The mosque was called Friday, congregational or biggest mosque of the city. After few decades, big city had some Friday mosques. In Persian, Friday translated as Jom'e and congregational is translated as jam'i. Masjid-e Jom'e is usual a Persian term to define a mosque. But in India they use also Persian terms however they call mosque jam'i masjid. In Arabia, Friday is translated as jum'a; to gather is translated as jama'a, and big means kabir. So in Arabia, mosque is called Masjid kabir. This issue 
points out the important requirement of a multilingual, structured and controlled vocabulary designed to cover the terminology of the Islamic historical field in architecture.

It is important to take into account that architectural tradition of preMuslim era based on other religion (Christian, Buddhism,...) and at the same time there were other religious monument (e.g. church, temple). Furthermore, Islamic architecture does not include only religious buildings but it includes also secular buildings as bazaar or houses [9]. Islamic Architecture will be one illusion, it was made by European scholars of 19th century to evaluate own culture.

Using architectural computing, especially semantic extraction, organisation and management, it introduces a newer way to think or re-think, providing a new point of view about the world architectural history, especially in our case, Islamic art, from the $21^{\text {st }}$ century's view.

In the paper, we investigate a new approach to categorize and to describe digital resources according to multi-facet cultural metadata sets. The Isfahan Islamic Architecture Database (IIAD) project over Internet demonstrates that this approach allows metadata optimization of the description of the cultural digital resources. Multi-faceted resource category management is integrated into a topic-maps based semantic management service part of the "myscoper" platform. This cooperative system aims at providing a web portal service in order to enable international multidisciplinary researchers and fellows to cooperate on research about historical architectural projects.

In Section 2, we introduce the problem issue and the states of the art in the field of historical and architectural semantic and metadata models. Then we present the topic-maps based metadata platform architecture, its different layers from data collection to semantic management and delivery in Section 3. Section 4 describes formally the multi-faceted resource category management. Then Section 5 overviews the case study related to Islamic Buildings of the city of Isfahan. Finally, Section 6 concludes and gives the direction of the future work.

\section{Issues related to historical and architectural} metadata models

\section{I. Multi-faceted resource categorization}

Information systems, like the brain, need also order so the users can understand their contents. A classification of cultural contents corresponds to a hierarchy of terms or controlled vocabularies. They are used for effective classification and searching for digital resources in bibliographical, digital databases and visual archives. Categories help the users to navigate inside the digital data collections [I]. By grouping the data by comprehensible categories, the users can quickly eliminate what is not

Tomoaki Okamura, Naoko Fukami, Charles Robert and Frederic Andres 
relevant or not interesting, and just look at the subject related to their interest. So, a multi-faceted categorization $[4,6,14]$ is typically end-user oriented as it enables a digital resource to be identified with more than one manner by different categories of classification.

Let us take AAT [39] as example along this article, a thesaurus of terms employed for the inventories and the indexes in the fields of art, architecture, artifacts and archives.

Figure $I$ is an example of multi-faceted classification of the Friday Mosque of Isfahan. It shows two viewpoints of classification, Facet I and Facet 2 classify the resource as a digital image and as a mosque respectively. Four-iwan mosque [17] was developed from the Seljuqid Iran from the early of the $12^{\text {th }}$ century and was spread from Egypt to India. Iwan is an open hall with large arched opening, originated in Iran since pre Islam era. Four-iwan means four open halls are constructed on the cross axis of the courtyard. Friday Mosque of Isfahan is a typical example of this predefined type as it is shown in [10]. This mosque has a complicated history. It was built in the $9^{\text {th }}$ century as hypostyle mosque. When the Isfahan became a capital of Seljuqid in the $\mathrm{I}^{\text {th }}$ century, Seljuqid Sultans added 2 domed halls, and renovated arcades to four iwan halls on the cross axis of the courtyard. And in the $15^{\text {th }}$ century under the Timurid, twin minaret was added at the main iwan on the south west direction. After 250 years in the late Safavid, the surface wall of courtyard was decorated by tiles with marble dado, and the vault of iwan was reconstructed with the muqarnas technique as it was studied in [28]. At the same time, digital images and mosques are global view. If we consider from the local point of view, components will be part of classification as iwan, minaret, muqarnas and arcade.

- Figure I: Digital photo of Friday Mosque of Isfahan.

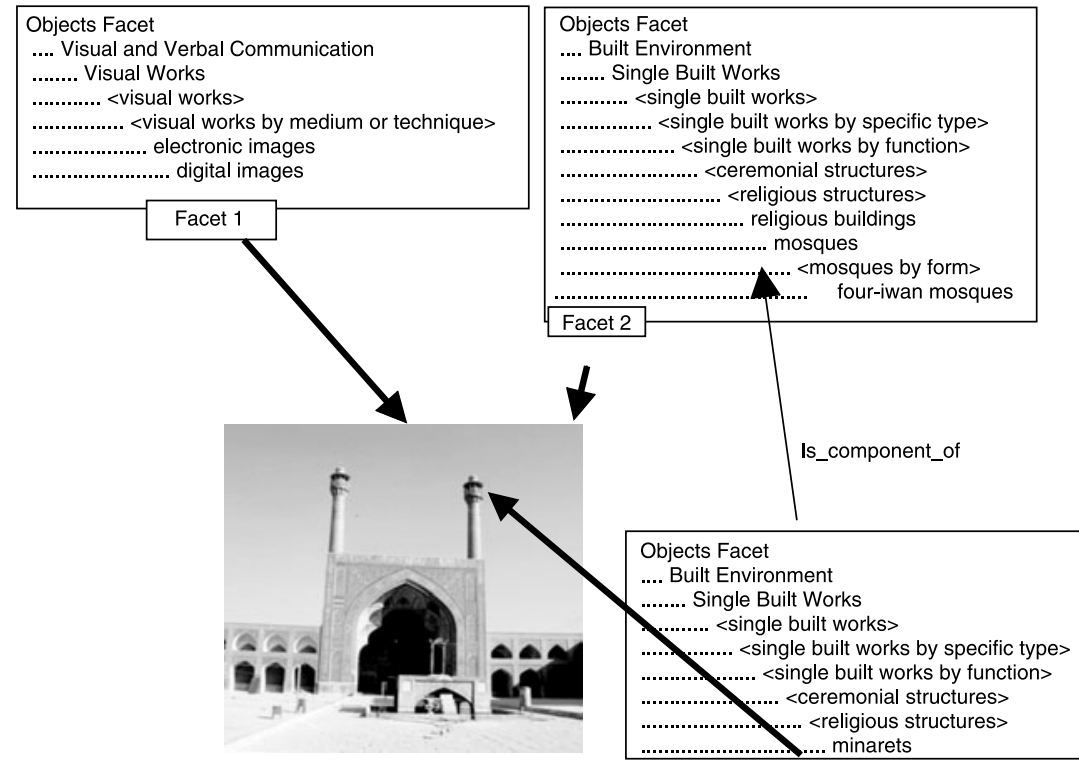

\begin{tabular}{ll|l} 
Digital Resource Semantic Management of Islamic Buildings Case Study on Isfahan Islamic & 359
\end{tabular} Architecture Digital Collection 
The multi-faceted classification [36] avoids the problem of traditional monolithic hierarchies considering that digital contents can be classified according to different dimensions (time, space) or viewpoints (usage, signification). Plural classifications are identified as categorization paths for the same resource using topic maps indexes. It is also important to combine an efficient way metadata and categorization in order to provide enriched descriptions of resources.

\subsection{Metadata and categorization}

Metadata standards reflect any information related to the content, the context, and the structure of resources which supports their effective use, including information which can facilitate their management, their access and their analysis. Also those metadata standards have been developed in parallel by different communities [15, 16, 19]. Also Isfahan Islam Architecture Database project is typically cross-disciplinary including architecture, history, human and social sciences. Content metadata of a digital resource about an historical building relates to what the resource contains or is about, and is intrinsic to a specific building. This category has been developed from several perspectives, based on the creator or the provider of the related metadata as it is shown in Figure 2. ECAI,VRA, MPEG-7, and OBJECT ID metadata sets facilitate the search on bibliographical information related to the resource (e.g. author, title, creation date, description, resource format etc.) from the content producer's perspectives. The ECAI core set includes the fifteen elements defined by Dublin Core and has been extended by the ECAI consortium to support time and space metadata. Though the metadata of $\mathrm{ECAl}$ are very generic, the descriptions are refined to be more specific by other metadata.

In addition, each digital image of historical building includes a lot of information, if we aim at physical characteristics and historical contents, $5 \mathrm{WIH}[38]$ has a key role to play in the contextual semantic part of our digital database. 5WIH uniformly describes a historical building with "Who, What, Where, When, How and Why". 5WIH that is applied to building-centric context can depict "a certain building user (Who) is", "in a certain location (Where)", "in a certain time (When)", "paying attention to a certain component of the building (What)", "given a certain physical and architectural view with material (How)", or "because of a certain problem or (Why)". For Islamic architecture experts, it enables to point out contextual characteristics to identify architectural objects or components. Furthermore, it also enables to describe the features of each component. Elements depict architectural building's contexts according to its environments. It enables to understand the size of space, the arrangement of space, used material, patterns, and geometrical features. Attributes provide the meta-data related to an element or relationship with other elements.

Tomoaki Okamura, Naoko Fukami, Charles Robert and Frederic Andres 
- Figure 2: Sample of Mosque Metadata Description.

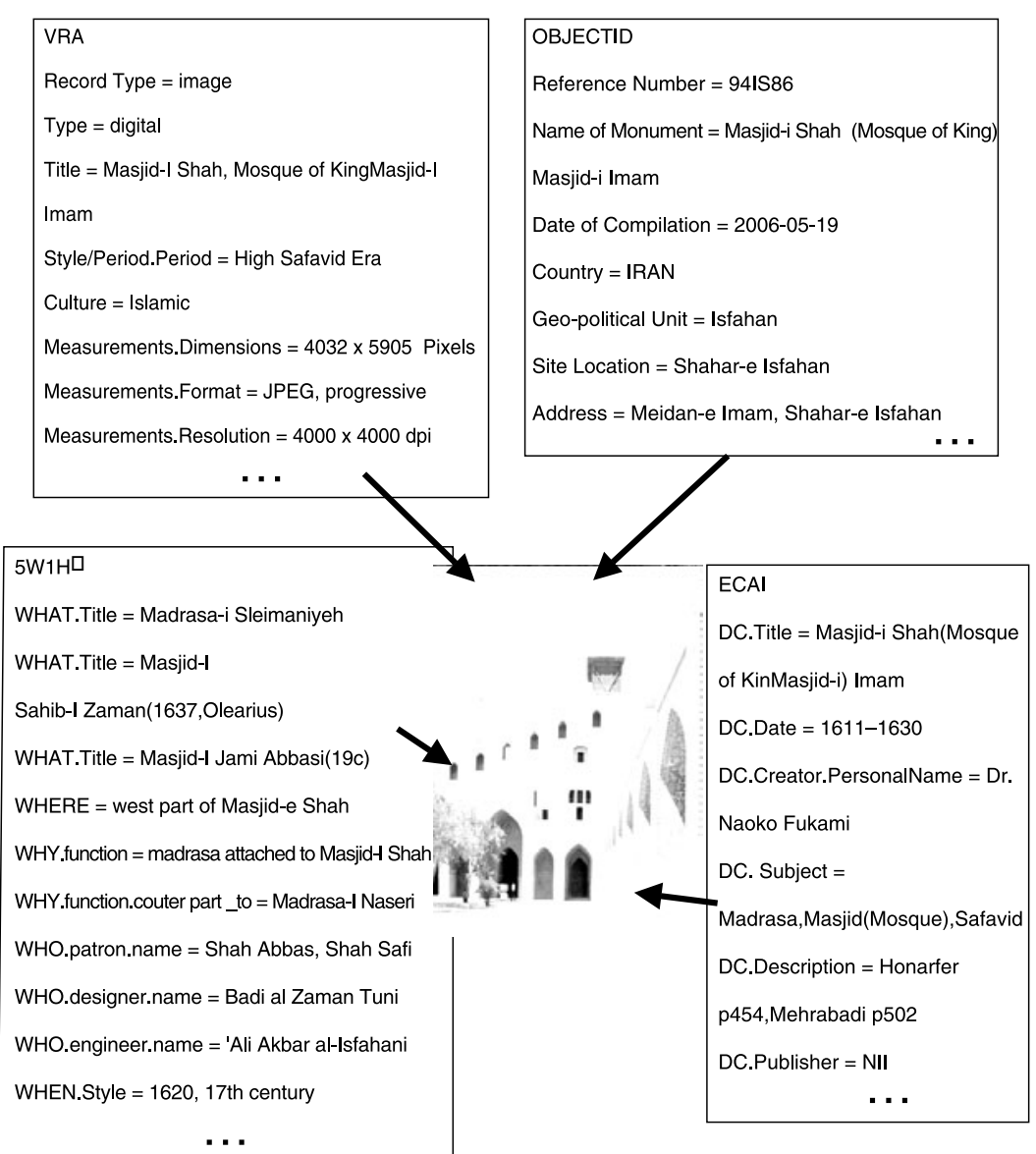

$\cdots$

"What" is also described as "title" or "name of monument" in some metadata description, but more varied means are for example usage or purpose. Public or private, religious or secular, etc.. "Where" is also included some metadata description as "location" or "address", again historical name of the place is effective, further more we add not only the geographical position but also detailed architectural components from the local view points. "When" is cited as "date" or "date of compilation", further the date of destruction and restoration are important, and the date be able to relate the periods or styles classified by architectural objects. "Who", particularly architect, artisan, patron, owner of building are not described in some metadata description, only the reference is adopted, but the person who connected the building tells detailed history. "Why" is very generic view to connect physical characteristics to historical contents, as the points of view from politics, economics, religion, nation or structure are needed. "How" introduces physical and architectural views that include materials, techniques, styles and patterns.

\begin{tabular}{ll|l} 
Digital Resource Semantic Management of Islamic Buildings Case Study on Isfahan Islamic & $\mathbf{3 6}$
\end{tabular} Architecture Digital Collection 
The metadata creation process, as a feedback from the IIAD project, has been split into three steps as following as it is shown in Figure 3:

I. Metadata creation during the Field data Collection (semi-automatic process)

- Documents

- Visual documents

2. Data analyses (automatic process)

- Visual documents

3. Multidisciplinary survey and comparison (semi-automatic process)

- Visual documents about buildings

- Visual documents about mosques.

The metadata management in the IIAD project relies on five standards: ECAI,VRA, MPEG-7, OBJECT ID and 5WIH.

\subsection{Bridge between metadata sets}

Each metadata set reflects some communities or specific fields [22, 24, 25, 26]. Hence, many similarity or differences of metadata description exist between metadata attributes. The attributes in some metadata sets are correlative with the attributes sets. Getty Research Institute has elaborated a horizontal Crosswalk [ I I] between the descriptors of metadata in the domains of culture, architecture, heritage and resource on line. This horizontal correspondence has been a first solution in terms of interoperability between the descriptors of metadata sets including DC, VRA, and ObjectID. Even such a kind of bridge [16, 19, 20, 2I] between the metadata sets facilitates the semantic interoperability and systematic conversion from one metadata set to another, the conversion is sometimes not sufficient. Relationship of specialization between attributes exists more than exact relationship.

\begin{tabular}{l|l|l|l}
\hline \multirow{2}{*}{$\begin{array}{l}\text { Resource } \\
\text { Vocument }\end{array}$} & Metadata support & End-users \\
\cline { 2 - 3 } $\begin{array}{l}\text { Visual document } \\
\text { Visual document } \\
\text { about a Building } \\
\text { Visual document } \\
\text { about a Mosque }\end{array}$ & & DC & Librarian \\
\cline { 2 - 3 } & & DC.VRA & Photo/web master \\
\cline { 2 - 3 } & & DC.VRA.MPEG7 & Semantic expert \\
\cline { 2 - 3 } & & & \\
\hline
\end{tabular}

4 Figure 3: Vertical Metadata Management according to end-users expertise and profile.

362 Tomoaki Okamura, Naoko Fukami, Charles Robert and Frederic Andres 
2.4. Links between multi-faceted categorization and topic maps-based metadata

Combining multi-faceted categorizations of resources and multiple cultural metadata sets in the Isfahan Islamic Architecture Database project was motivated by the recognition that: (I) no mapping has been done between metadata sets and resource classifications to enrich architectural databases, (2) there has been little research to provide enriched metadata collaborative framework based on multiple metadata sets and best practice results [3I, 34], and (I) several metadata standards (e.g. ECAI, VRA, OBJECTID) created in the past years will make benefit the IIAD project according to end-users' profiles and expertise, Language-dependent controlled vocabularies and cultural-depend thesaurus have been introduced to play an interpretive layer of semantics between the term entered by the user and the underlying cultural digital resource database. It also enables to preserve the quality of the user meaning and cultural background. Let us take an object can be annotated by various metadata sets. Figure 2 is an example annotated by the ECAI, VRA, ObjectID and 5WIH. Classifications of the same object are varied as each standard categorizes the object according to their own classification. Each metadata requires a different expertise as it is shown in Figure 3. Though there are many cultural metadata sets, no single metadata model exists for sufficiently managing interdisciplinary and inter-organization cultural contents. Hence, the multi-faceted resource category management and the mapping are highly required for the efficient data retrieval.

Providing multi-metadata management and Subject-dependent Categorization enables an enriched faceted metadata description to each resource as it is show in Figure 4. The multi-faceted topic maps-based metadata is realized by mapping the metadata sets into the thesaurus classification in order to be supported by various communities. The path in the AAT hierarchy enables to classify the attributes of each metadata set in the function of applicability.

Attributes of a mosque can be added at the leaf node to enrich each category by community content classification. There is no 'Mosque'related-metadata standard but it is one of major subjects in the Islamic architecture field.

As there is no simple one-to-one mapping corresponding to each other, the mapping is complex. We propose a new approach which maps multiple cultural metadata sets to cultural-dependent thesaurus avoiding the overlapping of attributes of different metadata sets. First, it enables the interoperability between multiple metadata sets according to one resource enriched category path. Second, it enables to provide a topic maps-based metadata management which is both language and cultural-dependent.

The Topic Maps-based metadata classification related to architectural objects and buildings shown in Figure 5 is based on the classification approach introduced in [7]. 


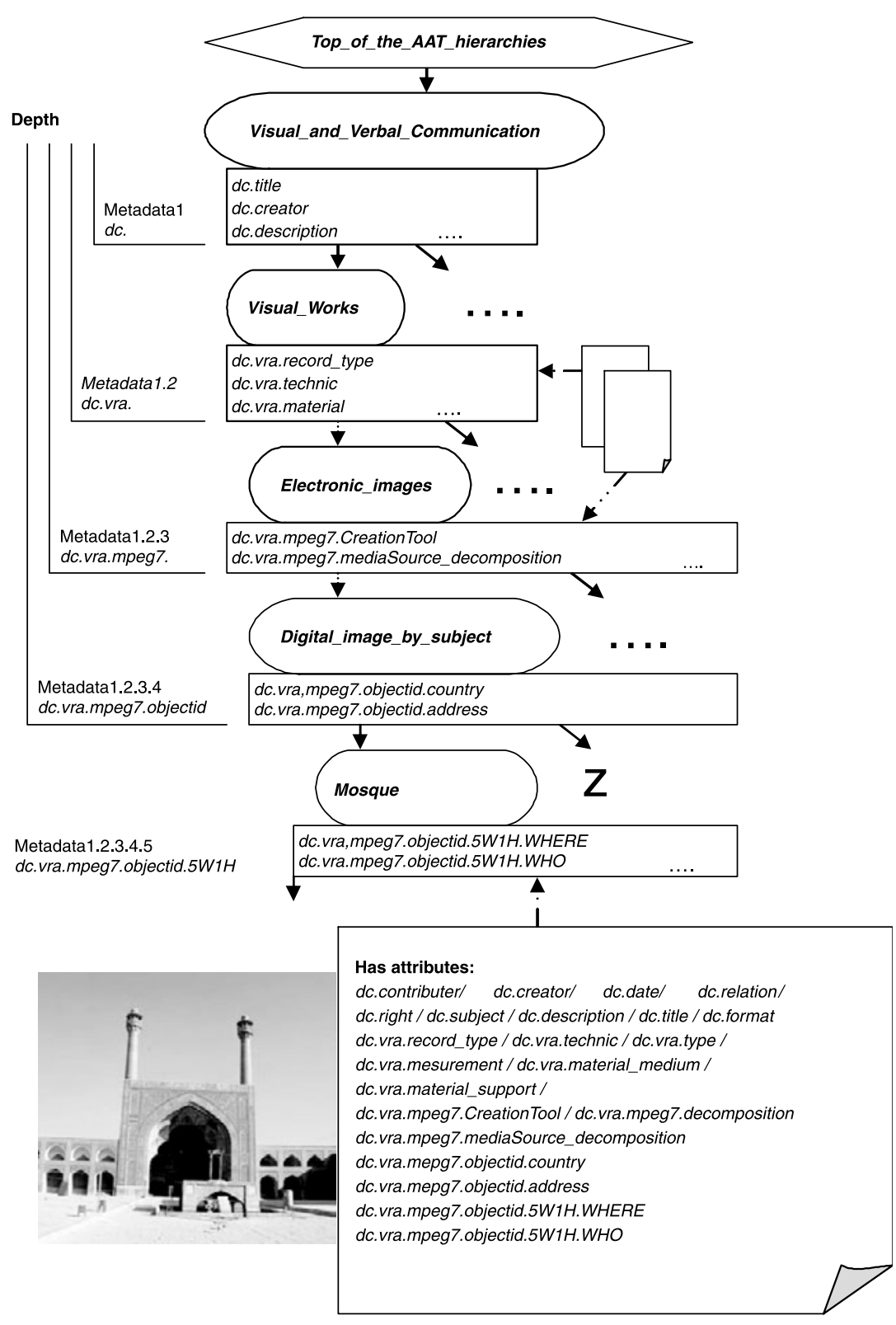

4 Figure 4: Topic Maps-hierarchical metadata description for the Friday mosque.

\section{Topic maps-based metadata management} architecture

We created a topic maps-based semantic management system in order to establish a multi-lingual systematic semantic inventory of digital contents for research and education purposes. It is part of the prototype which intends to provide a platform available over Internet to researchers, investigators, and those who are interested in sharing digital resources on Islamic historical buildings.

364

Tomoaki Okamura, Naoko Fukami, Charles Robert and Frederic Andres 
In this project, we gathered so-called historical monuments in Isfahan, which have been already described [3, 12, 18, 23, 27, 30, 32, 33, 35] or which have been discovered during the data collection process on that site [8]. We define monument an architectural building which can be religious, public building but also secular or private buildings. And from the view point of Isfahan's city history, we deal about civil engineering, for example roads, bridges, squares and gardens.

The topic maps-based semantic structure connects the mapping between multiple metadata sets and topics. No specific language is used as bridge language as the topic maps is language and culture-independent. The scope feature of the topic map structure provides the language and the cultural points of view.

In the following section, we will formalize the multi-faceted topic mapsbased metadata model from a multi-layer point of view in order to be able to avoid overlapping between metadata sets.

3.I. Topic maps for cultural resource and architectural computing

The first step to support resource algebra is the design of topic maps for cultural resource and cultural computing.

Figure 5: Topic Map-based Resource and Semantic Sharing Platform.

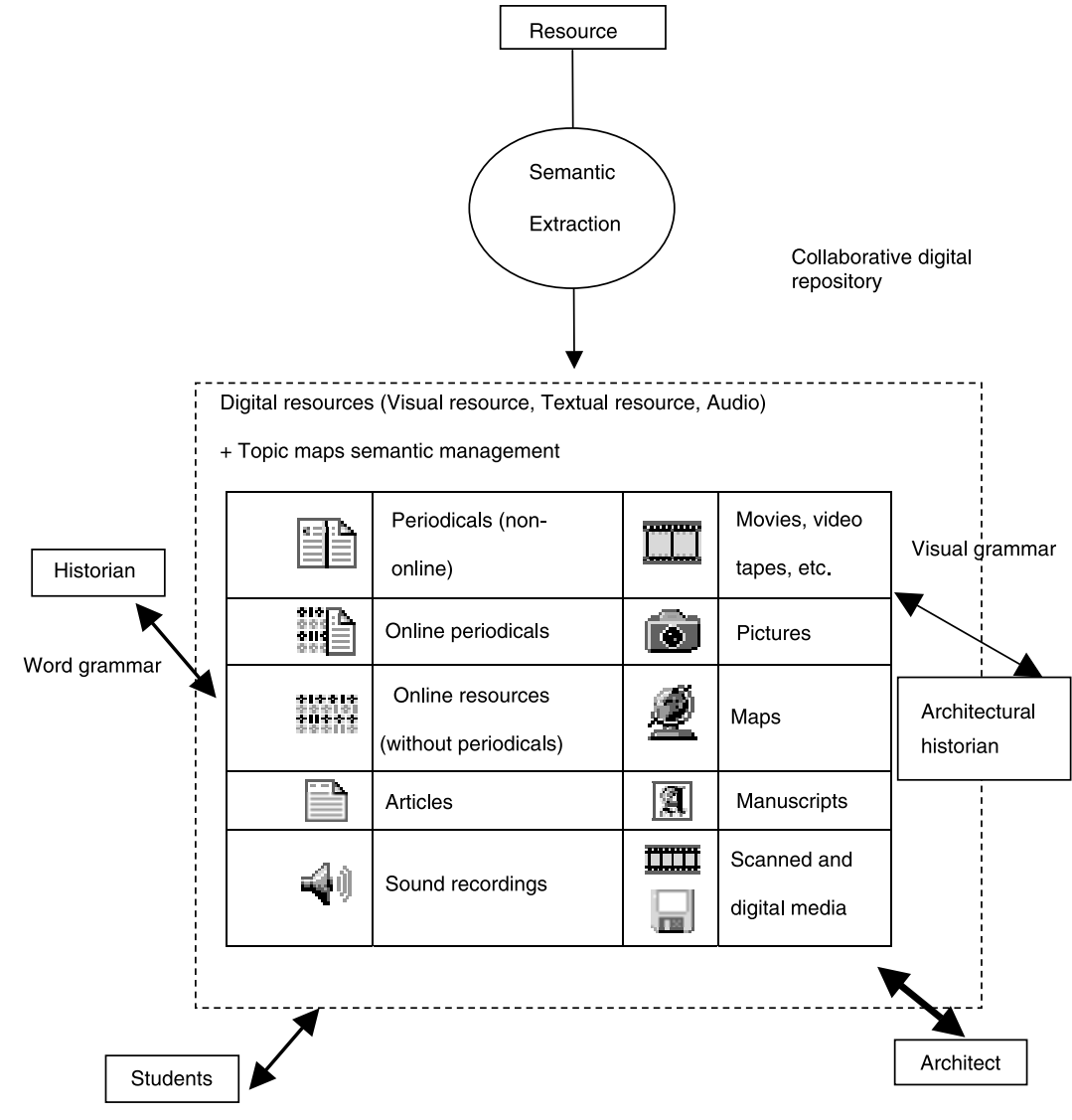

Digital Resource Semantic Management of Islamic Buildings Case Study on Isfahan Islamic 365 Architecture Digital Collection 


\subsection{The algebra}

The Resource algebra uses cultural domain underlying data types. Resource semantic type and functions in the topic maps are directly represented using the appropriate data type and functions supported by the resource algebra. This algebra follows two targets. First, it is the purpose of the semantic interface between scientists to reduce the semantic gap and to strength the metadata bridging between them.

Second, this high level semantic algebra facilitates the collaborative intersection of scientists using topic maps integrating high level semantics.

Let us remind the notion of many sorted algebra [39]. Such algebra consists of several sets of values and a set of operations (functions) between these sets. It consists of two sets of symbols called sorts (e.g. topic, pdf, rtf, Isi_sm) and operators (e.g. tm_transcribe, semantic_similarity); the function sections constitute the signature of the algebra. Second Order Signature [40] is based on two coupled many-sorted signatures where the top-level signature provides kinds (set of types) as sorts (e.g. DATA,

RESOURCE, SEMANTIC_DATA) and type constructors as operators (e.g. set) To illustrate the approach, we assume the following simplified many-sorted algebra, which is part of the TMblog project [29]:

Kinds DATA, RESOURCE, SEMANTIC_DATA,TOPIC_MAPS, SET

Type constructor

$\rightarrow$ DATA topic

-> RESOURCE pdf, rdt, htm, $\mathrm{xml}$, cvs, jpeg, tiff // resource document type

-> SEMANTIC_DATA Isi_sm, mpeg7_sm, dc_sm, vra_sm, cdwa_sm, ecai_sm, objectid_sm

// Semantic and metadata vectors

-> TOPIC_MAPS tm(topic maps)

TOPIC_MAPS ->SET set

Unary operations

$\forall$ Resource in RESOURCE, resource $\rightarrow$ sm: SEMANTIC_DATA,

tm tm_transcribe

$\forall$ sm in SEMANTIC_DATA sm $\rightarrow$ set(tm) semantic_similarity

The notion sm:SEMANTIC_DATA is to be read as "some type sm in SEMANTIC_DATA," and means that there is a typing mapping associated with the tm_transcribe operator. Each operator determines the result type within the kind of SEMANTIC_DATA, depending on the given operand resource types.

Binary operations

$\forall \mathrm{tm}$ in TOPIC_MAPS, $(\mathrm{tm})+\rightarrow \mathrm{tm}$ topicmaps_merging

$\forall$ sm in SEMANTIC_DATA, $\forall$ tm in TOPIC_MAPS,

366

Tomoaki Okamura, Naoko Fukami, Charles Robert and Frederic Andres 

predicate of type topic and returns a subset of the operand set fulfilling the predicate. From the implementation of view, the resource algebra is an extensible library package providing a collection of resource data types and operations for domain-oriented resource computation (e.g. cultural field).

The major research challenge will be the formalization and the standardization of cultural resource data types and semantic operations through ISO standardization.

\section{Topic-map based multi-faceted metadata support}

The Resource Categorisation Path is denoted RCP. Each node of the path (denoted $\alpha_{i}$ ) is associated with a topic name and a list of descriptors (denoted $\delta$ ), we defined RCP as

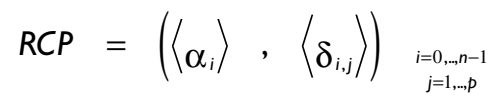

where $\delta_{i, j}$ represents the $j^{\text {th }}$ descriptor of the ith node, $n$ is the number of nodes and $p$ the number of descriptors in the ith node.

The whole Metadata Path Descriptor for the resource $r$ is defined for the complete branch of RCP as follows:

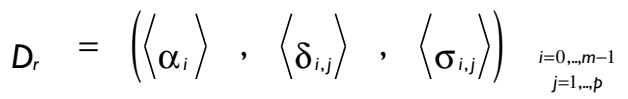

where $\sigma_{i, j}$ represents the $j^{\text {th }}$ descriptor value of the ith node, $m$ is the length of the path between the root of the RCP and the leave which references the resource (so called resource occurence).

We define a metadata set (denote $M$ ) as follows:

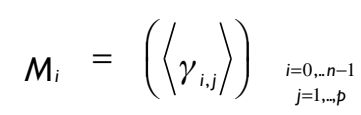

where $\gamma_{i, j}$ represents the $j^{\text {th }}$ attribute of the $\mathrm{i}^{\text {th }}$ metadata set.

For the $\mathrm{k}^{\text {th }}$ node of the RCP of the thesaurus $\lambda$, the resource $r$ has the resource descriptor defined as follows:

$$
D(\lambda)_{r}=\left(\left\langle\alpha(\lambda)_{k}\right\rangle,\left\langle\delta(\lambda)_{k, j}\right\rangle,\left\langle\sigma(\lambda)_{k, j}\right\rangle\right)_{j=1, \ldots p}
$$

\begin{tabular}{ll|l} 
Digital Resource Semantic Management of Islamic Buildings Case Study on Isfahan Islamic & 367
\end{tabular} Architecture Digital Collectio 
where $p$ represents the number of attributes

Property I:

For a leave node $f$, we define

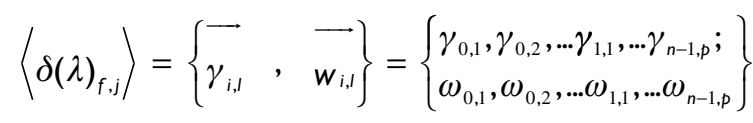

where $\omega_{i, j}$ is the binary variable that indicates whether or not the $j^{\text {th }}$ attribute $\gamma_{i}$ of the $i^{\text {th }}$ medatadata set appears in the descriptor $\delta$ of the $\mathrm{f}^{\text {th }}$ node.

Let us use the AAT as our main reference as English Thesaurus. The AAT is a culture-dependent structured vocabulary that can be used to improve access to information relating to fine art, architecture, decorative arts, archival materials, and material culture. It contains more than 133,000 terms, descriptions, bibliographic citations, and other information about concept.

The AAT Resource Categorization Tree is defined by

$$
\operatorname{RCP}(\mathrm{AAT})=\left(<\alpha(\mathrm{AAT})_{\mathrm{i}}>,<\delta(\mathrm{AAT})_{\mathrm{i}, \mathrm{j}}>\right)
$$

where $\delta_{i, j}(A A T)$ represents the $j^{\text {th }}$ attribute of the $i^{\text {th }}$ node, $n$ is the number of nodes and $P$ is the number of attributes of the $i^{\text {th }}$ node.

\section{I. Semantic optimization}

We developed the topic maps-based metadata management along the ISO/IEC I3250.

As shown in Figure 6, we introduce an extract of AAT Topic maps regarding regarding mosque classification.

$$
\begin{aligned}
& \begin{array}{l}
\text { AAT } \\
9-0 \text { Objects Facet }
\end{array} \\
& \text { Objects Facet } \\
& \text { B Single Built Works } \\
& \text { Pingle Built Works } \\
& \text { o } \square \text { <single built works by general type } \\
& \text { i- }- \text { ssingle built works by specific type } \\
& \text { <single built works by condition } \\
& \text { «single built works by condition } \\
& \text { - «single built works by design } \\
& \text { 9. - \& s single built works by function } \\
& \text { D. exhibition buildings } \\
& \text { eagricultural structures: } \\
& \text { Q. - }-9 \text { <eremonial structures } \\
& \text { a- }-9 \text { <funerary structures } \\
& \text { P. - - < religious structures: } \\
& \text { i- } \square \text { religious buildings } \\
& \varphi-\square \text { mosques } \\
& \text { P } \square \text { <mosques by form: } \\
& \square \text { four-iwan mosques }
\end{aligned}
$$

Tomoaki Okamura, Naoko Fukami, Charles Robert and Frederic Andres

Figure 6: Topic Maps classification of mosque in AAT. 
Furthermore, the complete structure used for metadata management in the IIAD project is composed by the two main classes; Metadata type class defining multi-metadata set and metadata class for the AAT thesaurus hierarchy. The Metadata type class defines hierarchically the attributes of metadata sets and composed of the sub-classes [Figure 7] according to the following classification; Administrative, Contents, Descriptive, Physical, Conservation, Products, Technique and Usage.

We have been using the AAT_thesaurus class for projecting the description of metadata sets in its sub-classes according to the type of resource. The projection will be detailed in the following section.

Figure 7: Metadata Type Schema.

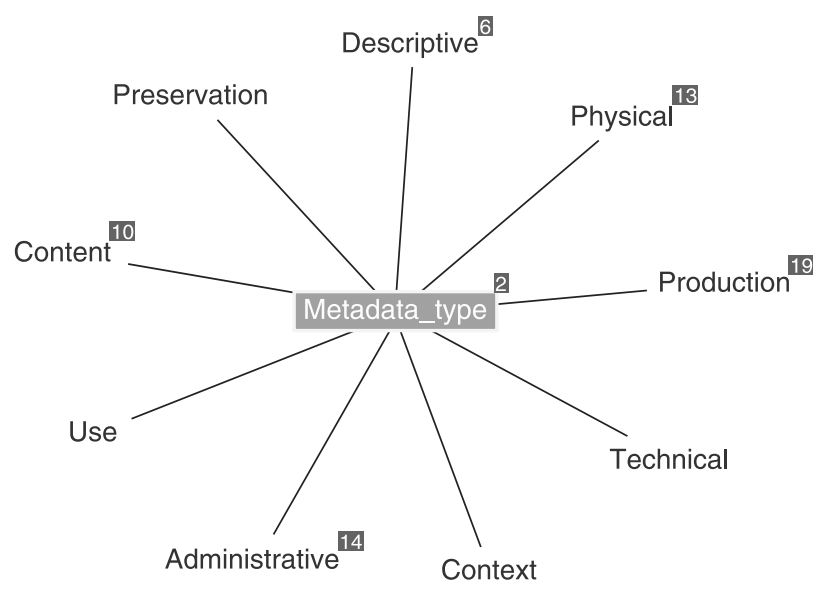

4.2. Usability study and discussions

Working with a real data set has been important to construct a first version of the multi-faceted metadata classification. The multifaceted metadata has been created by mapping the attributes in the AAT hierarchy avoiding overlapping similar attributes. As part of the IIAD collection, we selecting mosque collection for this study to point out and to address three issues related to the usage of attributes from multiple metadata sets: (I) Similarity between attributes, (2) Specialization between attributes, and (3) Attributes categorization by subject.

Similarity between metadata attributes

As it has been overviewed in Section 2, metadata standards for cultural resources have been developed in parallel by different communities from various perspectives. The bridge of Getty as a « Crosswalk » provides horizontal correspondences between metadata attributes. Controlling the equivalence between metadata attributes is an important issue as in this horizontal approach, the similarity mapping is rarely. An attribute can belong to several metadata sets and has the same significance.

\begin{tabular}{ll|l} 
Digital Resource Semantic Management of Islamic Buildings Case Study on Isfahan Islamic & 369
\end{tabular} Architecture Digital Collection 
$\mathrm{RI}$ : let us define an attribute a belonging to two metadata descriptions DI and D2., its domains of value are equivalent or exactly similar, the rule of attribute usage is defined as following:

$$
D_{1} \cdot a \equiv D_{2} \cdot a \quad \rightarrow D_{1} \cdot a
$$

Specialization between metadata attributes

- Case of similar attribute's name

$R 2: \forall b$ an attribute of $D_{1}$ is a specialization of an attribute $b$ of $D_{2}$

$$
\begin{aligned}
& D_{1} \cdot b \stackrel{\text { specialises }}{\longrightarrow} D_{2} \cdot b \quad \underset{\text { specialises }}{\longrightarrow} D_{2} \cdot c \wedge D_{2} \cdot c \stackrel{\text { specialises }}{\longrightarrow} D_{2} \cdot D_{1} \cdot b \\
& D_{1} \cdot c \stackrel{ }{\longrightarrow} \cdot c \quad \Rightarrow D_{3} \cdot D_{2} \cdot D_{1} \cdot c
\end{aligned}
$$

- Case of a different attribute's name

R3: $\forall b$ an attribute of $D_{2}$ is a specialization of an attribute $c$ of $D_{1}$

$$
D_{2} \cdot b \stackrel{\text { is_a_specialisation }}{\longrightarrow} D_{1} \cdot c \quad \Rightarrow D_{1} \cdot D_{2} \cdot b
$$

The attribute $b$ will be used in the classification path

R4: case of an attribute which exists in only one metadata set

$$
D_{3} . d \quad \Rightarrow D_{1} \cdot D_{2} \cdot D_{3} . d
$$

Where $D_{3}$ is the metadata set specializing $D_{1}$ and $D_{2}$

If we generalize, when $D_{n}$.d is the most specialized attribute, we get the rule:

$$
D_{n} \cdot d \Rightarrow D_{1} \cdot D_{2} \cdots D_{n} \cdot d
$$

\section{Conclusion}

This article presented a new way to share resources and related semantics according to metadata sets. We overviewed the Topic maps-based MultiFaceted Metadata Model. It aims to improve collaborative semantic management in digital cultural archives. It mainly regarding the way to manage cultural ontology based metadata as it allows the optimization of the description of the cultural resources.

We also introduced a categorization of digital resources based on a topic maps-based metadata hierarchy. The multiple metadata sets are vertically mapped as topic maps thesaurus (e.g. AAT hierarchies).

The multi-faceted metadata model of resources provides an enriched multiple metadata set. It takes an advantage to link the metadata sets between institutes or communities. It is the first approach enabling to realize a single hierarchical metadata set covering the cultural resource.

We demonstrated an implementation in the case of the cultural resources involving the metadata sets ECAI,VRA, OBJECT ID and MPEG-7.

Tomoaki Okamura, Naoko Fukami, Charles Robert and Frederic Andres 
The system is extensible to multi-lingual management, as we have been developing multi-facet metadata model and a thesauri classification independently.

The current IIAD includes 900 high resolution $(4000 \times 4000)$ building images with related multi-facet multi-metadata classification. The collection will be collaboratively extended and adding contextual semantics.

\section{Acknowledgements}

We like to thanks NII for this International Cooperation support and the Ministry of Education, Science and Technology for the support to the Geomedia project and the IIAD project (Isfahan Islamic Architecture Database) project.

\section{References}

I. Aldershoff, F., Salden Alfons H., lacob Sorin M., Kempen M., Supervised Multimedia Categorization, in Proc. SPIE Vol. 502I, p. 100-III, Storage and Retrieval for Media Databases 2003; Minerva M. Yeung, Rainer W. Lienhart, Chung-Sheng Li; Eds., Jan 2003.

2. Blair, S., and M. Bloom J., 1994 The Art and Architecture of Islam, I250-1800 New Haven, Yale University Press pp.348 ISBN-13: 978-0300058888 ASIN: 0300058888

3. Chardin, J. I7II, Voyages de Mr. Le Chevalier Chardin, en Perse, et autres lieux de l'Orient.,Vol.2. Amsterdam: Jean Louis de Lorne.

4. Colyer, G., Ishii, K., Hunter, J., Mapping between Dublin Core and JPX (JPEG 2000) Metadata, ISO/IEC JTCI/SC29/WGI N2736, I5 October, 2002.

5. Ettinghausen, R., and Grabar, O., 1987 The Art and Architecture of Islam, 650-1250, New York, Viking Penguin.

6. Frew, J., and Janée, G., A Comparison of the Dublin Core Metadata Element Set and the Alexandria Digital Library Bucket Framework. Technical report 2003.

7. Fujisawa, S., and Andres, F., Multi-facet Category for Cultural Digital Resources, ICDEW, p. |227, 2 Ist International Conference on Data Engineering Workshops (ICDEW'05), 2005.

8. Fukami, N., Madrasas at Isfahan, From Architectural Features and their Distributions, Memoire of Institute of Oriental Culture, University of Tokyo, publication No 137 1999-03, p. 257-294, ISSN 05638089.

9. Fukami, N., Safavid House in Isfahan, Memoire of Institute of Oriental Culture, University of Tokyo, publication No I39, 2000-03, p. I52-206, ISSN 0563889.

10. Gardieri., E. 1972-3, 1984 Isfahan: Masgid-I guma, 3 vols, Rome, ISMEO.

II. Godby, C. J.,Young, J. A., Childress, E., Repository of Metadata Crosswalks, in D-Lib Magazine, December 2004, Volume 10 Number 12.

12. Golombek, Lisa \& D. Wilber, The Timurid Architecture of Iran \& Turan, 2 vols, Princeton Pronceton University Press, 1988.

13. Necipoglu, G., Framing the Gaze in Ottoman, Safavid, and Mughal Palaces, Ars Orientalis Pre-Modern Islamic Palaces, American Oriental Society, Vol. 23 1993, Pp. 303-342, ISSN 057I-137I.

14. HandschuhI S. Staab, S., and Studer, R., Leveraging Metadata Creation for the Semantic Web with CREAM, In R. Kruse et al., KI 2003 - Advances in Artificia Intelligence. Proc. of the Annual German Conference on Al, volume 282I, pp. 19-33. Springer, Berlin, September 2003.

Digital Resource Semantic Management of Islamic Buildings Case Study on Isfahan Islamic Architecture Digital Collection 
15. Haynes, D., Metadata for Information Management and Retrieval, Facet Publishing London, 2004, xiv + I86 pages, hardback, ISBN I 856044890.

16. Hill, L. L., and Janée, G., The Alexandria Digital Library Project: Metadata Development and Use, (2004). Metadata in Practice (Diane I. Hillmann and Elaine L. Westbrooks, editors) (Chicago: American Library Assocation): $117-138$

17. Hillenbrand, R., Islamic Architecture, Edinburg University Press, 1994, pp. 173-25 I, 508-524.

18. Honarfar, L., 1965-6 Gangine-ye atar-e tarixi-ye Esfahan, Esfahan.

19. Hunter, J., Working Towards MetaUtopia, - A Survey of Current Metadata Research, Library Trends, Organizing the Internet, Edited by Andrew Torok, 52(2), Fall 2003

20. Hunter, J., A Proposal for the Integration of Dublin Core and MPEG-7, ISO/IEC JCI/SC29/WGII M6500, 54th MPEG Meeting, La Baule, October 2000.

21. Hunter, J., Combining the CIDOC/CRM and MPEG-7 to Describe Multimedia in Museums, Museums on the Web 2002, Boston, April 2002.

22. Hyvönen, E., Salminen, M., Kettula, S., and Junnila, M., A Content Creation Process for the Semantic Web, Proceedings of OntoLex 2004, 2004.

23. Herdeg, K., Formal Structure In Islamic Architecture of Iran and Turkestan, Rizzol International publisher, New York, 199I/03,ISBN 97808478I0482, pp. 53.

24. Junnila, M., Hyvönen, E., and Salminen, M., Describing and Linking Cultural Semantic Content by Using Situations and Actions. Developments in Artificial Intelligence and the Semantic Web - Proceedings of the 12th Finnish AI Conference STeP 2006, October 26-27, 2006

25. Lagoze, C., and Hunter. J., The ABC Ontology and Model, In Journal of Digital Information, Volume 2 Issue 2 Article No. 77, 200 I-I I-06.

26. Linda, L. Hill, Janée, G., Dolin, R., Frew, R., and Larsgaard, M., Collection Metadata Solutions for Digital Library Applications, Journal of the American Society for Information Science (JASIS) 50(13) (November 1999): I I69-II8I.

27. Alemi, M.,"The Royal Gardens of the Safavid Period_Types and Models". Gardens in the Time of the Great Muslim Empire, pp. 72-96, Leiden, A. Petruccioli ed, 1997.

28. Haneda, M., The Character of the Urbanization of Isfahan in the Late Safavid Period, in Charles Melville Ed., Safavid Persia, London \& New York, IB Tauris \& Co, 1996 369-387.

29. Rajbhandari, S., Andres, F., Naito, M., and Wuwongse, V., Topic Management in Spatial-Temporal Multimedia Blog, the Ist IEEE International Conference on Digital Information Management (ICDIM 2006), Bangalore, India, December, 6-8, 2006 Pp. 8I-88, ISBN: I-4244-0682-X

30. Redaxan, S.: Map of Isfahan by Sultan Seyyed Reza Khan in 1302 H., Teheran, 1984

31. Shabajee, P., Miller, L., and Dingley, A., Adding Value to Large Multimedia Collections Through Annotation Technologies and Tools: Serving Communities of Interest, in proceedings of Museums and the Web 2002, Boston, USA.

32. Babaie, S., Safavid Palaces at Isfahan - Continuity and Change (1590-1666) Insitute of Fine Arts, New York University, 1993, PhD Dissertation.

33. Tavo, B., VII - I4.5, Beisplile Islamicher Stadte, Das Safavidische Isfahan, 1989.

34. Weinstein, P. C., Ontology-Based Metadata: Transforming the MARC Legacy, in the proceedings of Digital Libraries 98, Pittsburgh, USA, Pp. 254-263.

35. Wilber, D., The Architecture of Islamic Iran: The II Khanid Period, Princeton Pronceton University Press, 1955.

36. Yee, K., Swearingen, K., Li, K., and Hearst, M.: Faceted metadata for image search and browsing, in Proceedings of the SIGCHI conference on Human factors in

372 Tomoaki Okamura, Naoko Fukami, Charles Robert and Frederic Andres 
37. Zhang,Y., Semantic-Based Visual Information Retrieval, IRM Press (December II 2006), 368 Pp, ISBN- I0: 1599043718.

38. Kuo, P.-.., Aoki, T., Yasuda, H., MPEG-7 based dozen dimensional digital content architecture for semantic image retrieval services, in: IEEE '04. 2004 IEEE International Conference on e-Technology, e-Commerce and e-Service, 28-3I March 2004, 517-524.

39. http://www.getty.edu/research/conducting_research/vocabularies/aat/.

Tomoaki Okamura, Naoko Fukami

Institute of Oriental Studies

University of Tokyo

7-3-I Hongo, Bunkyo-ku

Tokyo I I3-0033 Japan

okamura4575@aol.com

fukami@ioc.u-tokyo.ac.jp

Charles Robert

LORIA, Campus scientifique, BP 239

54506, Vandoeuvre les Nancy, France

abiodun-charles.robert@loria.fr

Frederic Andres

NII, 2-I-2 Hitotsubashi, Chiyoda-ku

Tokyo I0I-8430, Japan

andres@nii.ac.jp

Digital Resource Semantic Management of Islamic Buildings Case Study on Isfahan Islamic 373 Architecture Digital Collection 
Chapter $10 \quad 16 / 7 / 07 \quad 12: 31$ pm Page 374 


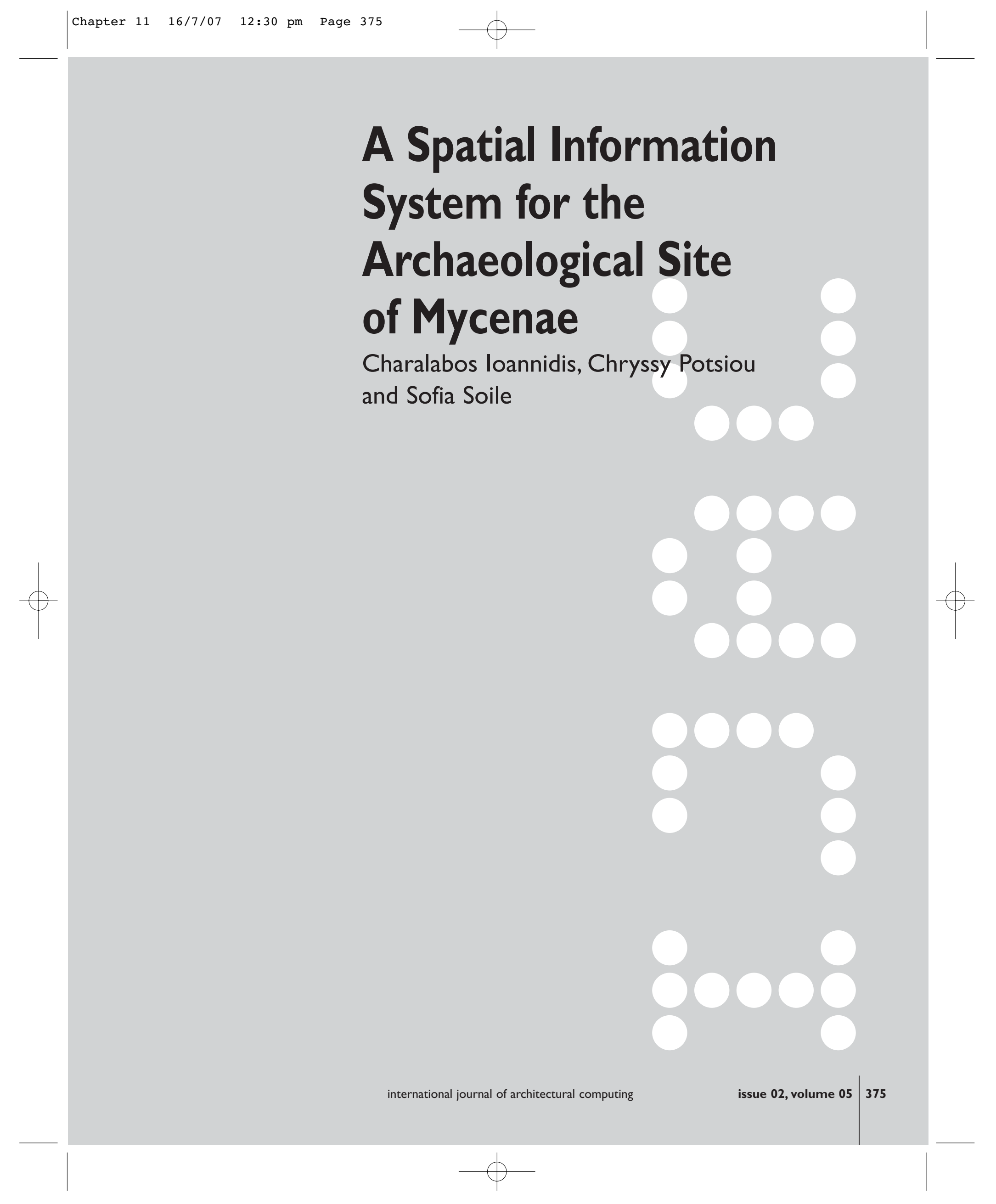

